\title{
Hemodynamic impact of positive end expiratory pressure in right cardiac performance
}

Keywords: mechanical ventilation, atelectatic alveoli, thoracic computed tomography, intrathoracic pressure, airway pressure, tidal volume

Abbreviations: MV, mechanical ventilation; Rap, right atrial pressure; Paw, airway pressure; Vt, tidal volume; Pms, mean systemic pressure; RV, right ventricle; RVEDP, right ventricular end diastolic pressure; PAC, pulmonary artery catheter; PVR, pulmonary vascular resistance; TLC, total lung capacity; FRC, functional residual capacity; $\mathrm{PAO}$, decreased alveolar oxygen tension; $\mathrm{LV}$, left ventricular

\section{Introduction}

The lungs and the cardiovascular system are closely interrelated and intimately intertwined. Both must be able to assure adequate gas exchange and peripheral blood distribution in order to keep metabolic demands. Either in respiratory failure or when the oxygen requirements of respiratory muscles increase beyond the capacity of the circulatory system to meet equilibrium between oxygen consumption and delivery, it is necessary to use mechanical ventilation (MV).

It is well known that inappropriate use of MV may induce lung injury and enhance lung damage caused by the underlying disease. The evaluation of respiratory mechanics and thoracic computed tomography has taught us that overdistention and opening and closing of atelectatic alveoli are the most important factors that promote ventilator induced lung injury. The protective role of low tidal volumes has been established; however, the optimal adjustment of positive end expiratory pressure remains uncertain, because it has not been possible to establish a balance between optimal recruitment and avoidance of alveolar overdistention and deleterious hemodynamic effects. ${ }^{1}$

The hemodynamic effects induced by ventilation depend on the changes in lung volume and intrathoracic pressure (Pith). In contrast with spontaneous breathing, during positive-pressure ventilation, both lung volume and Pith increase, phenomenon that contributes to explain the magnitude of the hemodynamic impact that characterizes this kind of ventilation. Although airway pressure (Paw) determines lung volume and Pith, this relationship also depends on both lung and chest wall compliance. When lung compliance is reduced, such as in acute lung injury, the extent to which Paw increases Pith is less than when lung compliance is normal, therefore hemodynamic consequences are ameliorated. The opposite is true when chest wall compliance is reduced. ${ }^{2,3}$

From a hemodynamic standpoint we must distinguish between the cardiovascular response to PEEP, and tidal volume (Vt). While PEEP remains during inspiration and expiration, thus maintaining its cardiovascular effect during the whole respiratory cycle, $\mathrm{Vt}$ induces only cyclic lung volume and airway pressure increments. In the first case, compensatory responses by means of the activation of neurovascular reflexes and the transdiaphragmatic transmission of
Volume 12 Issue 5 - 2019

\author{
Vinko Tomicic Flores \\ Internal Medicine-Intensive Medicine,Andrés Bello National \\ University, Chile
}

Correspondence: Vinko Tomicic Flores, Internal MedicineIntensive Medicine, Head of Respiratory Intensive Care Unit, Indisa Clinic, Andrés Bello National University, Santiago, Chile, Email vtomicic@gmail.com

Received: September 30, 2019 | Published: October 29, 2019

Pith occur. Interventions such as intravascular volume expansion and vasoactive drug administration can attenuate hemodynamic impact, especially in hypovolemic patients. ${ }^{4}$

\section{Venous return physiology}

As established by Guyton, venous return is promoted by a forward pressure, the mean systemic pressure (Pms) - which is the equilibrium pressure in the systemic vessels under non flow conditions - and is impaired by a backward pressure, the right atrial pressure (Rap).

In absence of obstruction in the vena cava, the systemic venous return must overcome the Rap so that the blood returns to the heart. Thus, at less Rap more venous return. This relationship is almost linear until Rap reaches zero, and further reductions will not increase venous return because the vena cava collapses. At the same time, Rap is the driving force that fills the right ventricle (RV) during diastole. Therefore, at high Rap, preload to the RV will be greater and, with its normal function, the cardiac output will increase. However, the relation between Rap, venous return and cardiac output must be seen as a dynamic response to the changes in pressure gradients throughout the cardiovascular circuit, because Rap can only be kept low if the venous return is rapidly extracted by means of an efficient $\mathrm{RV}^{4}$.

\section{The effects of PEEP on right atrial and right ventricular preload}

If assumed that the atmospheric pressure surrounds cava vein, right atrium and RV, any Rap higher than zero would hinder venous return with the same force with which it would drive RV filling. During PEEP ventilation this can change, since RV filling is determined by the difference between Rap and pericardial pressure (Ppc), known as the right atrial transmural pressure (PtmRA).

When PEEP is applied, part of it is transmitted to the pericardium, reducing the PtmRA and right ventricular preload, finally decreasing the right ventricular cardiac output. This means that the venous return 
still faces the same resistance, but the right cardiac output decreases. For example, if the Rap is $8 \mathrm{~cm} \mathrm{H}_{2} \mathrm{O}$ and the $\mathrm{Ppc}$ is $0 \mathrm{~cm} \mathrm{H}_{2} \mathrm{O}$, the PtmRA will be $8-08$, where Rap=PtmRA. Under these conditions, the driving pressure for RV filling and the pressure opposing the venous return are the same.

If $10 \mathrm{~cm} \mathrm{H}_{2} \mathrm{O}$ of PEEP are applied, approximately $5 \mathrm{~cm} \mathrm{H}_{2} \mathrm{O}$ will be transmitted to the pericardium. If the Rap is $8 \mathrm{~cm} \mathrm{H}_{2} \mathrm{O}$, the PtmRA will be $8-5=3 \mathrm{~cm} \mathrm{H}_{2} \mathrm{O}$. That is, to maintain RV preload at the level prior to PEEP application, Rap must increase to $13 \mathrm{~cm} \mathrm{H}_{2} \mathrm{O}$ so that PtmRA remains in $8 \mathrm{~cm} \mathrm{H}_{2} \mathrm{O}$. Therefore, right cardiac output depends on the PtmRA (intrathoracic component), and the pressure opposing the venous return is given by the Rap (extrathoracic component).

The Rap increase induced by PEEP is proposed as the primary mechanism for venous return reduction. This phenomenon is due to a reduction in the Pms - Rap gradient. In order to compensate this effect and maintain right ventricular filling, Pms must increase.

Studies in healthy dogs have shown that the application of PEEP increases Pms so that the Pms - Rap gradient remains constant. Three mechanisms have been proposed to explain the increase in Pms induced by PEEP application: a) PEEP displaces blood from the central to the peripheral circulation, increasing the systemic blood volume; b) the caudal diaphragm displacement increases intra-abdominal and hence splanchnic bed pressure ${ }^{5}$, and c) by inducing neurovascular reflexes, PEEP may increase systemic vascular tone. ${ }^{6}$. In contrast, patients with hypovolemia or adrenergic blockade could be unable to increase Pms, which may lead to the sudden hypotension eventually observed when positive pressure ventilation is instituted or after applying PEEP. 5,6

Other studies have shown that both the lower and upper cava venous returns decrease during PEEP ventilation, suggesting an increase in the central venous resistance, which induces a reduction in the right ventricular end diastolic volume. ${ }^{7}$

Even though PtmRA has received scarce attention, a correct evaluation of fluid administration while applying PEEP could be better performed from a PtmRA approach, which requires Ppc estimation. It is very important to elucidate that the right ventricular end diastolic pressure (RVEDP) is as important as PtmRA, because both determine RV preload. On the other hand, an increased RVEDP, as in pericardial tamponade, may indicate a reduction in RV compliance instead of an increased preload. Considering that under normal conditions RVEDP is close to $0 \mathrm{~cm} \mathrm{H}_{2} 0$, its increase can be used to estimate pericardial pressure. In the clinical scenario, RVEDP can be obtained during the insertion of the pulmonary artery catheter (PAC). Finally, PtmRA gives us a more accurate evaluation of the right ventricular filling pressure, and as such determines the cardiac output in both ventricles.

\section{Effects of PEEP on right ventricular performance}

Besides the right ventricular preload reduction described above, PEEP can increase the afterload of this ventricle due to its effects on the pulmonary vascular resistance (PVR). This happens because PEEP, by modifying the lung volume - main determinant of the blood flow at this level - can distort the pulmonary capillaries and produce paradoxical effects on the PVR and the pulmonary blood volume. In order to understand the effects of ventilation on both parameters, the anatomical relationship of the normal pulmonary circulation and its interaction with the alveoli and the pleura should be first considered. ${ }^{8}$
According to the pressure that surrounds the pulmonary vessels, they are divided into two categories: alveolar and extra-alveolar. The alveolar vessels are those adjoining the alveoli, and thus subjected to alveolar pressures. That is, as the alveoli distend during inspiration, the alveolar vessels are compressed, increasing resistance to blood flow. This effect becomes more pronounced as the lung inflates from the functional residual capacity (FRC) to the total lung capacity (TLC).

The extra-alveolar vessels, distant from the alveoli and adjacent to the pleura, are submitted to the pleural pressure (Ppl). That is why they are considered extra-pulmonary structures and as such, have a perivascular pressure that is equal to or slightly more negative than Ppl. In contrast with the alveolar vessels, here, as the lung volume enlarges, the elastic recoil of the pulmonary interstitium increases, acting as a suspension mechanism that dilates the extra-alveolar vessels. This phenomenon decreases the extra-alveolar vascular resistance by increasing its capacitance during inspiration and is more prominent moving from the residual volume to the FRC. ${ }^{9}$ This occurs in both spontaneous and positive pressure ventilation. The combined effect of lung expansion on resistance in alveolar and extra-alveolar vessels is a "U" shaped curve in which total PVR rises when lung volume is increased or decreased from FRC. Whenever PEEP-induced alveolar recruitment is achievable, its application can restore FRC so that tidal ventilation can be kept in the area of lowest PVR.

When lung volume decreases below FRC (insufficient PEEP) PVR can increase. This effect can be secondary to extra-alveolar vessels collapse or to decreased alveolar oxygen tension $\left(\mathrm{P}_{\mathrm{A}} \mathrm{O}_{2}\right)$ inducing hypoxic pulmonary vasoconstriction. The result would be an increase in right ventricular afterload. PEEP up to a certain level can induce an end expiratory lung volume expansion that increases extra-alveolar vessels capacitance reducing PVR and right ventricular overload. On the other hand, excessive PEEP to exceed the normal FRC can also increase PVR inducing right ventricular overload. This effect can also be seen in patients with obstructive lung disease who develop intrinsic PEEP.

The flow through the pulmonary vessels is generated by the gradient between pulmonary arterial pressure that impels blood, and pulmonary venous pressure that impedes this flow. In zone 3, Pvp opposes pulmonary blood flow; however, during inspiration it can be exceeded by alveolar pressure (Palv), which increases zone 2 proportion at the expense of zone 3. Strictly, during inspiration, the real pressure that opposes blood flow in zone 2 is transpulmonary pressure or lung distending pressure. Consequently, as tidal volume increases and in order to open the lung valve, RV must elevate pressure gradient during isovolumetric contraction. Through doppler ecocardiography measurements, some authors have observed a reduction in mean pulmonary artery blood flow when the tidal volume is doubled (320 to $650 \mathrm{~mL}$ ). It must be emphasized that in order to maintain its ejection fraction against afterload increased, as observed when condition 2 prevails, RV resorts to the Starling mechanism so that its expansion becomes its principal compensatory mechanism..$^{10,11}$

Another mechanism, through which PEEP can compromise the right ventricular function, is myocardial ischemia. When applying PEEP, pulmonary vascular resistance, free wall tension, and RV afterload increase, and that leads to augmented myocardial oxygen demands. Furthermore, PEEP has a direct effect on the coronary blood flow, probably by compressing the coronary vessels against the epicardium. Besides the effect on the coronary vascular resistance, 
PEEP can reduce coronary flow by decreasing the left ventricular (LV) output. ${ }^{12-14}$

\section{Acknowledgments}

None.

\section{Conflicts of interest}

The authors declare there are no conflicts of interest related to the article.

\section{Funding}

None.

\section{References}

1. Kavanagh BP. Target in mechanical ventilation for ARDS. En: Slutsky AS, Brochard L, Eds. Mechanical Ventilation. Update in intensive care and emergency medicine. Berlin Heidelberg: Springer-Verlag, 2004. p. 287-300.

2. Pinsky MR. Recent advances in the clinical application of heart-lung interactions. Curr Opin Crit Care. 2002 8(1):26-31.

3. Scharf SM, Ingram RH. Effects of decreasing lung compliance with oleic acid on the cardiovascular response to PEEP. Am J Physiol. 1977;233(6):H635-H641.

4. Fessler HE, Brower RG, Wise RA, et al. Effects of positive endexpiratory pressure on the gradient for venous return. Am Rev Respir Dis. 1991;143(1):19-24.

5. Jellinek H, Krenn H, Oczenski W, et al. Influence of positive airway pressure on the pressure gradient for venous return in humans. $J$ Appl Physiol. 2000;88(3):926-932.
6. Selldén H1, Sjövall H, Wallin BG, et al. Changes in muscle symphatetic nerve activity venous plasma catecholamines and calf vascular resistance during mechanical ventilation with PEEP in humans. Anesthesiology. 1989;70(2):243-250.

7. Dhainaut JF, Devaux JY, Monsallier JF, et al. Mechanisms of decreased left ventricular preload during continuous positive pressure ventilation in ARDS. Chest. 1986;90(1):74-80.

8. Lai-FooK SJ. Perivascular interstitial fluid pressure measured by micropipettes in isolated dog lung. $J$ Appl Physiol. 1982;52(1):9-15.

9. Howell JB, Permutt S, Proctor DF, et al. Effect of inflation of the lung on different parts of the pulmonary vascular bed. J Appl Physiol. $1961 ; 16: 71-76$

10. Vieillard-Baron A, Loubieres Y, Schmitt JM, et al. Cyclic changes in right ventricular output impedance during mechanical ventilation. $J$ Appl Physiol. 1999;87(5):1644-1650.

11. MEBAZAA A, KARPATI P, RENAUD E ET AL. Acute right ventricular failure-from pathophysiology to new treatments. Intensive Care Med. 2004;30(2):185-196.

12. Fessler HE, Brower RG, Wise R, et al. Positive pleural pressure decreases coronary perfusion. Am J Physiol. 1990;258(3 Pt 2):H814-H820.

13. Luecke T, Pelosi P. Clinical review: Positive end-expiratory pressure and cardiac output. Crit Care. 2005;9(6):607-621.

14. Çoruh B, Luks AM. Positive End-Expiratory Pressure. When More May Not Be Better. Ann Am Thorac Soc. 2014;11(8):1327-1331. 